\title{
The Role and Trend of Information and Communications Technology Towards a Pervasive Healthcare System
}

\author{
Oluwadara J. Odeyinka, Federal University of Technology Owerri, Owerri, Nigeria \\ Opeyemi A. Ajibola, University of Lagos, Lagos, Nigeria \\ Michael C. Ndinechi, Federal University of Technology Owerri, Owerri, Nigeria
}

\begin{abstract}
Technological innovations have played a significant role in the modern healthcare system. However, with increasing rate of human population relative to the available medical personnel and facilities, a sudden outbreak of epidemic diseases and its resultant spread into a pandemic may pose a risk to the wellbeing of human populace. Thus, state-of-the-art innovations in communication engineering and technology is able to provide a support mechanism to healthcare domain. This paper analyzed pillars and paradigms in information and communications technology (ICT) deployable in the healthcare domain and estimates a compliance index based on the subset in which the ICT pillars and paradigms are decomposed. The analysis revealed that technological advances in the areas of robotics and internet of things (IoT) paradigm presented a potential support mechanism for healthcare domain. Finally, the paper concluded with insight into the implementation of these technologies and other supporting pillars and paradigms towards a pervasive healthcare system, enlisted recommendations, and future directions.
\end{abstract}

\section{KEYWORDS}

Compliance Index, Healthcare Domain, ICT Paradigms, ICT Pillars, Internet of Things, Technological Innovations 


\section{INTRODUCTION}

Over the last decade, communication engineering especially in the area of wireless networks had achieved landmark success and emerged as the key technology for enabling the mobile revolution. To this end, series of generations of wireless communication networks have emerged, paving way for smart delivery of information from one point to another. The speed, capacity, robustness and accuracy of wireless communication keeps improving everyday thereby enhancing its suitability in a wide range of applications such as: E-healthcare, disaster management, disease control, smart diagnosis, etc. Thus, the implementation of Information and Communications Technology (ICT) in healthcare system cannot be over-emphasized. ICT healthcare paradigms is sub divided into: e-health, mobile health, smart health, ubiquitous health, personalized health and pervasive health (Giuseppe et al., 2018). Varshney, (2007) defined pervasive healthcare as healthcare to anyone, at anytime and anywhere by removing locational, time and other restraints while increasing both coverage and the quality of healthcare. As a result of tremendous increase in human population, traditional healthcare is unable to accommodate everyone's need (Prabha et al., 2017). However, to control a sudden outbreak of epidemic infection and the infection spreading into a pandemic may pose a serious challenge to local and global health organizations. Thus, to address such challenge, there is a need for an effective healthcare monitoring system that is scalable, real-time and smart. A smart system that will report in realtime, analyze incoming data and provide actionable insight. Thus, harnessing emerging technology in communication engineering can remotely monitor the health condition of in-hospital patient and the entire populace. Also, the starting points of an epidemic can be early detected and awareness widely created through applications on hand-held mobile devices and other sensing instruments.

Technological advances in the area of Wireless Sensor Networks (WSNs), Artificial Intelligence (AI), Nano-medicine and other supporting technology in the area of Information and Communications Technology (ICT) have the potential to improve the study and observation of patients response to treatment by allowing vital sign (data) to be collected and inputted automatically into patients care record and used for realtime triage, correlation with healthcare record and long-term observation (Myung et al., 2003). These technological advances can also permit remote monitoring for chronic illness and disease control, collection of information for medical database; this could enhance comprehensive studies across human population and enable medical practitioners to study the effect of a sudden outbreak of epidemic diseases and would affords the opportunity to plan timely intervention programs. Therefore, the alignment of Information and communications technology with human capability can enhance a quick healthcare delivery in Hospitals and Medical centers. 


\section{ARCHITECTURE OF A TYPICAL COMMUNICATION ENGINEERING SYSTEM}

In communication engineering, there is a functional principle that ensured the transmission and reception of information at an acceptable degree of fidelity. The information source is coded, processed and transmitted through a channel to a signal/ Information receiver where it would be decoded to obtain the original information. Figure 1 is a block diagram to illustrate the concept.

Figure 1. Block diagram of information and communications system

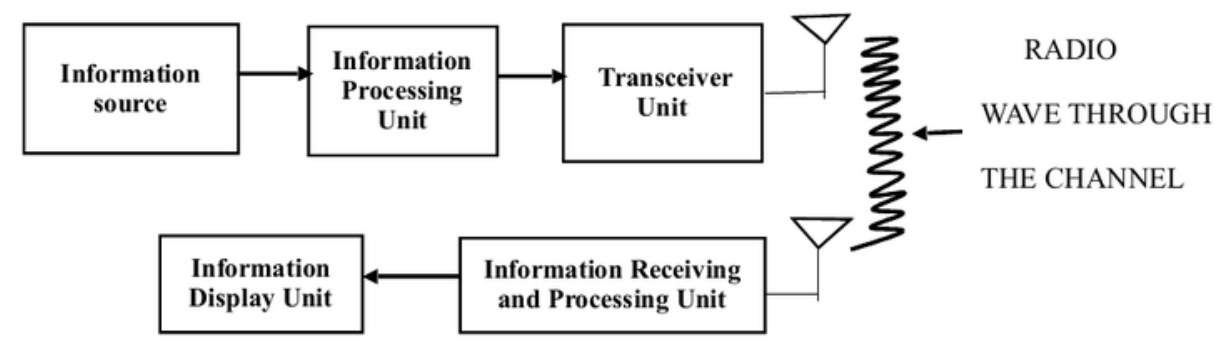

- Information Source: This is the data to be processed and transmitted. It exist as analogue signal, such information may include: Sound, images/pictures, video signals, physical parameters like temperature, pressure etc.;

- Processing Unit: This is sometimes referred to as storage/processing unit. The information (usually analogue) is received through its input device (possibly a sensor), converted into a digital form using Analogue-to-Digital Converter (ADC). The digital signal is then forwarded to the transceiver unit for transmission;

- Transceiver Unit: This is a device capable of transmitting and receiving radio frequency signal. The transceiver is connected to an antenna to transmit through the channel the digital signal in the form of electromagnetic wave (Radio wave) by altering the wave characteristics such as Frequency, Amplitude, Phase or a combination of these characteristics; a process called modulation;

- Information Receiving and Processing Unit: At the receiver unit, the antenna receives the weak signal, the signal is then amplified, demodulated and decoded. Thereafter the signal is displayed in its original form on the information display unit.

\section{ICT BASED HEALTHCARE FRAMEWORK AND REQUIREMENTS}

The four main personnel involved in healthcare are: Doctors, Patients, Hospitals and Government or Research Personnel. They determine activities such as: Disease prevention and control, medical diagnosis and treatment of illness, hospital management, medical and technological research. The smartness of these relevant 
activities in healthcare delivery is dependent on the speed and accuracy with which medical services is being delivered. ICT based healthcare architecture is defined based on: Services, Medical devices, Connectivity, System management (mgt), Applications and End users. Figure 2 illustrates details of this framework. However, requirements of ICT based healthcare can be classified into: Functional and nonfunctional requirements. In functional requirements, specific services of a healthcare architecture based on application of deployed components are addressed. Contrariwise, non-functional requirements are not specific. This refers to characteristics on which the quality of healthcare system can be determined (Prabha et al., 2017). Typically, the non-functional requirement is sub-divided into performance and ethical requirements. Performance requirement being of paramount importance to this subject, is categorized as hardware and software requirements which defined metrics as: Power efficiency, quality of service, reliability of the system, inter-operability, connectivity, speed, accuracy, ambient intelligence and memory.

\section{ICT PILLARS AND PARADIGMS SUPPORTING HEALTHCARE DOMAIN}

Based on the requirement of ICT based healthcare system, there are a number of technology in the area of data and information communication serving as Pillars and Paradigms for the sustainability of healthcare domain Table 1 is a list of ICT Pillars, Paradigms and ICT based healthcare paradigms.

Based on the general trends outlined by the ICT-based healthcare paradigms, the overall ICT eco-system have been decomposed in four subset: Sensing, Processing, Communication and Actuation (Giuseppe et al., 2018). The compliance index of the ICT Pillars and Paradigms on Table 2 is measured based on the decomposed subsets. Considering the subsets of ICT eco-system and the varieties of available technologies, it should be noted that these subsets are not dis-joint. Similarly, several Pillars and Paradigms can interoperate, paving way for a more robust, efficient and smart system.

Figure 2. Framework of ICT based healthcare

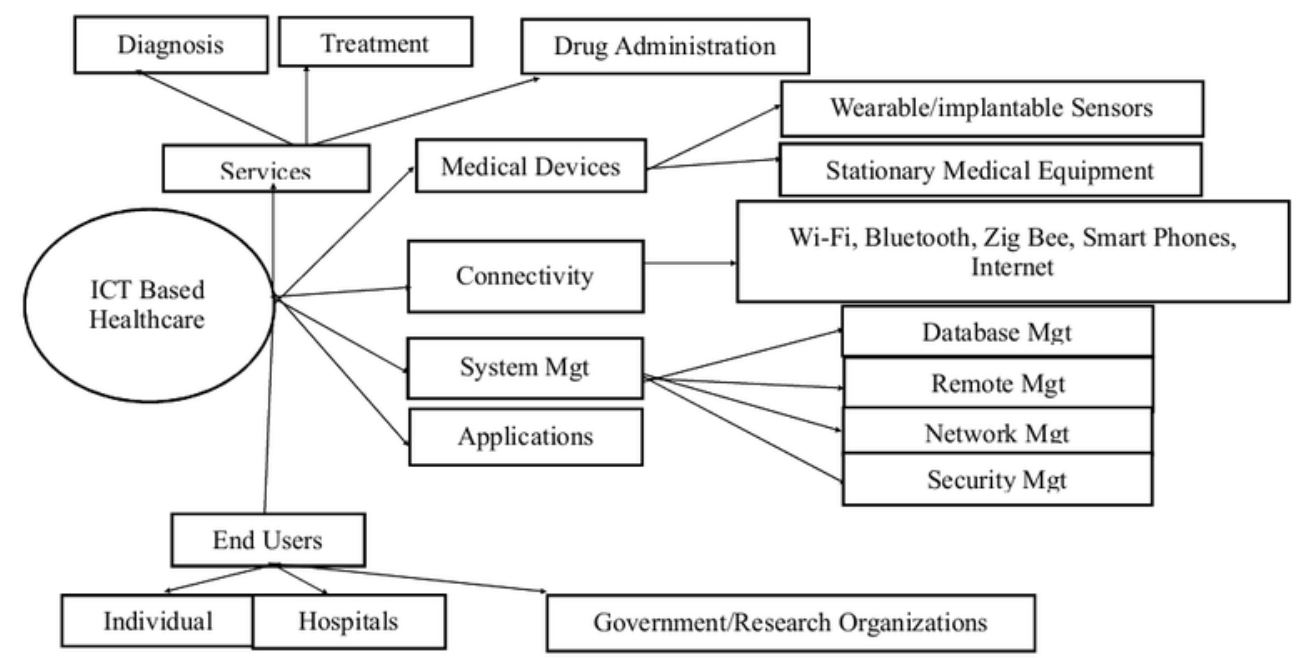


Table 1. ICT pillars/paradigms and ICT-based healthcare paradigms

\begin{tabular}{|l|l|l|}
\hline \multicolumn{1}{|c|}{ ICT Pillars } & \multicolumn{1}{|c|}{ ICT Paradigms } & ICT-Based Healthcare Paradigms \\
\hline Artificial Intelligence (AI) & Cloud Computing & E- Health \\
\hline Big -Data Analytics & Cognitive Computing & Mobile Health \\
\hline Personal Devices & FOG Computing & Personalized Health \\
\hline Robotics & Internet of Things & Pervasive Health \\
\hline Sensing Technologies & Machine-to-Machine & Smart Health \\
\hline Social Media & Mobile Cloud & Ubiquitous Health \\
\hline Smart Devices & Mobile Edge Computing & \\
\hline Virtualization Techniques & & \\
\hline Wireless Sensor Networks & & \\
\hline Wearable Devices & & \\
\hline Wired Networks & & \\
\hline Wireless Networks & & \\
\hline Wireless Body Area Networks & & \\
\hline 3D Printing & & \\
\hline
\end{tabular}

Sources: Content within the table are extracted from Google search engine

The concept behind the Pillars and Paradigms having common elements in some cases and the subsets; sensing, processing and communication operating in tandem to the essential principle of communication engineering enhances the applicability of these Pillars and Paradigms towards an effective healthcare delivery system. The subset; actuation (enabling a system to actively respond to its environment based on information or signal received) embedded in some of the Pillars and Paradigms and other prospective ones would necessitate their requirement in providing solutions to the challenges in modern day healthcare system. The definitions below briefly explain some notable ICT Pillars and Paradigms and their potential role such as: Support for clinical and medical database, intelligent diagnosis, medical devices, and internet connectivity for healthcare.

\section{Artificial Intelligence}

This is a specialized field of computer science that is concerned with the development of algorithms for a computing system to be able to imitate human thought processes such as; reasoning, learning and self-correction. With the application of technologies such as Artificial Intelligence (AI), diagnosis and treatment of diseases has become more intelligent. The accuracy of AI diagnosis results exceeds that of human doctors (Polat\&Gunes, 2008), (Dhar\&Ranganathan, 2015), (Esteva et al., 2017). The most outstanding and representative products in the field of clinical decision support system is IBM's Watson (Shuo et al., 2019) an intelligent cognitive system providing an optimal solution through in-dept analysis of all clinical data. Through the implementation of clinical decision support system, medical personnel can give expert and precise advice 
with the aid of algorithms to improve the accuracy of diagnosis, thereby enabling patients to receive immediate and appropriate medical attention.

\section{Big Data Analytics}

Big data is a set of data which a computer cannot capture, manage and process within an acceptable limit (Chen et al., 2014). Big data analytics describe a new era of technologies designed to economically retrieve valuable data from a very large volumes of different variety of dataset by enabling the high-speed capture and analysis. Thus big data characteristics can be: Volume (large volume), Variety (Various types and structure of data), speed (quick creation) and Value (Great value but very low similarity).

\section{Cloud Computing}

This is a convenience enabling model, providing on-demand access to a shared pool of configurable computing resources such as: Services, networks, server, storage, and applications that can be rapidly released or made available with minimal managerial effort or service provider interaction (Srinivas et al., 2012).

\section{Cognitive Computing}

This is one of the newly emerging ICT Paradigm composed of intelligent computing methodologies and utilization of computational intelligence through autonomous inferences and perceptions that duplicate brain mechanisms (Yingxu, 2009).

\section{Internet of Things (IoT)}

This is a global network infrastructure, dynamic in nature with self-configuring capabilities based on standards. IoT Paradigm serve as a host for a community of inter-operable communication protocols where Virtual and Physical 'things' are identifiable, utilizes intelligent interfaces, and are seamlessly embedded into the information network (Van Kranenburg, 2008). IoT utilizes a technology that interacts with anyone, anything, anywhere and anytime. Figure 3 illustrate the concept of IoT interaction. Thus, in a precise definition, Internet of Things is an Internet of three things: Human to Human, Human to Things (machine and devices) and Things to Things, interacting anywhere and at any time through the Internet.

\section{Edge Computing}

Edge computing in telecommunication is often referred to as Mobile Edge Computing (MEC) or Multi- Access Edge Computing. It is a new paradigm of substantial computing with storage resources. The storage resources also referred to as cloudlet, Fog nodes or micro-data center are placed at the edge of the internet so that computing happens in close proximity to data sources. The enriching edge computing resources extends to both downstream data for cloud services and upstream data for Internet of Things services (Shi \&Schahram, 2016). 


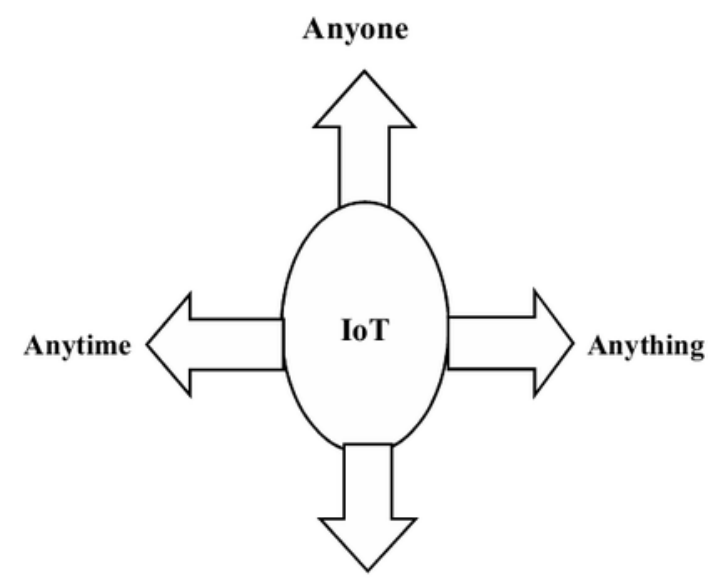

Anywhere

\section{FOG Computing}

This is a newly emerging ICT Paradigm utilized for the Internet of Things (IoT). Fog computing enhances the extension of Cloud computing and services to the edge of the network (Bonomi et al., 2012). It equally provides to end users; data, storage, computation and application similar to that of Cloud computing ( $\operatorname{Rad} \&$ Shareef, 2017). Fog computing differs from Cloud computing in the following: (i) Close proximity to end-users. (ii) It provides enhancement for mobility. (iii) It provides support in dense geographical distribution.

\section{Mobile Cloud Computing (MCC)}

This is defined as 'a rich mobile computing technology that leverages unified elastic resources of varied clouds and network technologies towards unrestricted functionality, storage and mobility to serve a multitude of mobile devices anywhere, anytime through the channel of Ethernet or Internet regardless of heterogeneous environments and platforms based on the pay-as-you use principle' (Sanaei et al., 2013) and (Mojtaba et al., 2016). The main objective of MCC is to enable execution of Rich Mobile Application on plethora of mobile devices with rich user experience.

\section{Robotics}

This is an interdisciplinary research area at the interface of information engineering, computer science and engineering, mechanical and electronic engineering. Robotics involves design, construction, operation and use of Robots ('Robotics,' 2020). The objective of robotics is to design intelligent based machine that can substitute for human and replicate human actions. 


\section{Social Media}

Social forum through the Internet media is a potential empowering, engaging and educating tool for healthcare providers and consumers. Social media like Tweeter, personal blogging, wikis and other platforms can revolutionize the healthcare domain as patients can inexpensively turn to a social network groups to connect other patients who are battling the same diseases, share advice, connect to or recommend a medical personnel thereby reducing anxiety about a certain ailment.

\section{Soft Computing}

This is an important tool to perform several computing operations that includes: fuzzy logic, neural networks, models, appropriate reasoning and evolutionary algorithm such as genetic algorithm and simulated annealing. The soft computing allows to incorporate human knowledge effectively, deal with uncertainty, imprecision and learns to adapt to unknown or changing environment for better performance (Panda, 2014).

\section{Wireless Sensor Networks (WSNs)}

A wireless sensor network is a self-configured and infracstructureless networks of wireless sensor nodes to measure physical or ambient parameters and raw data such as: Temperature, pressure, acoustic, motion etc., processing the raw data and communicating to the resource management center or Sink for necessary action. As Wireless Sensor Networks (WSNs) have become mature enough in nature as: Self organization, mobility and control among others, they have become one of the major technology deployable in healthcare environment. Indeed, WSNs for healthcare have emerged in recent years, driven by the need to collect data about physical, physiological, psychological, cognitive and human behavioral process. Some of the merits of WSNs in healthcare are: Supporting interfaces for the disabled, automated patient monitoring, drug administration and diagnosis, tele-monitoring of patients, physiological data and tracking the activities of Doctors and health workers within the hospital.Some notable examples of wireless sensors applicable in healthcare domain are: Electrocardiograph (ECG/EKG), Electroencephalograph (EEG) and Electromyography (EMG) sensors for measuring electrical signal of Heart, Brain and Muscles respectively, thermal sensor for measuring human body temperature, glucose sensor for monitoring the amount of glucose in the blood and a host of other sensors.

\section{Wireless Body Area Networks (WBANs)}

Wireless body area networks encompasses an in-vitro (attached to the body) and an in-vivo (Implanted in the body) intelligent devices, capable of establishing wireless communication networks. There are some variations that include the word 'wireless' and or the word 'sensor'. Thus, wireless body area networks and wireless body area sensor networks are used interchangeably. WBANs comprises of the following:

1. Wireless Sensor Nodes: These are sensors that responds to physical parameters, gather data on them and possibly process or report the acquired data; 
2. Actuator Nodes: These are nodes that responds relative to information or data received from sensor nodes and interact with users. Actuator nodes can be a simple fixed electronic system or a soft-ware based system;

3. Body Control Unit: This is a smart device also referred to as Body gateway which aggregates all the information or data received by the sensor nodes and inform the end-user through an external gateway, an actuator or an information display unit.

Data communication protocol designed for Wireless Body Area Networks encompasses communication between the sensors attached to the body and communication from body gateway to a resource management center connected to the cloud.

\section{Wearable and Smart Devices}

Wearable devices are commonly applicable in healthcare domain due to their potentials in data acquisition and analysis. Information acquired from wearable devices can be useful in providing insight to physiological variations and condition of patients. This will enable medical experts to effectively monitors patients health conditionand response to treatment, dispense appropriate medication, schedule a timely visit and propose rehabilitative solution. Primarily, wearable devices are sensor embedded devices capable of obtaining physiological data e.g. health bands. However, technological advances is transforming wearable devices into smart devices embedded with micro-processor for performing local processing of sensed data and data transmission module to forward the data to a resource management center. Thus, a smart device is a context-aware; mobile or immobile electronic devices with selfcomputational capability and able to establish a wire or wireless connection to other devices for the purpose of data or information exchange. Devices such as smartphones, smart watches and smart google glasses may fit into this category.

\section{COMPLIANCE INDEX OF ICT PILLARS/PARADIGMS IN ICT BASED HEALTH CARE PARADIGM}

Based on the ICT Pillars/Paradigms on Table 1 supporting the ICT based healthcare paradigms and the ICT eco-system being divided into four subsets (Giuseppe et al., 2018): Sensing capability, Processing capability, Communication capability and Actuation capability.

Let $\mathrm{U}=\{$ Set of ICT Pillars/Paradigms in healthcare Domain $\}$

Set $\mathrm{S}, \mathrm{P}, \mathrm{C}$ and $\mathrm{A}$ are subsets of $\mathrm{U}$.

$\mathrm{S}=\{$ ICT Pillars/Paradigms with Sensing capability $\}, P=\{$ ICT Pillars/Paradigms with Processing capability $\}, \mathrm{C}=\{$ ICT Pillars/Paradigms with Communication capability $\}$ and $\mathrm{A}=\{$ ICT Pillars/Paradigms with Actuation capability $\}$. Ç- define intersection of sets 
- $\quad \mathrm{S}$ only, P only, C only and A only illustrates ICT Pillars/Paradigms with a single capability and are indicated on Table 2 as having a compliance index ' 1 ';

- SÇP, SÇC, SÇA, PÇC, PÇA and CÇA all defined ICT Pillars/Paradigms with two capabilities and are indicated on Table 2 as having a compliance index ' 2 ';

- SÇPÇC, SÇCÇA and PÇCÇA all defined ICT Pillars/Paradigms with three capabilities. If any, and are indicated on Table 2 as having a compliance index ' 3 ';

- SÇPÇCÇA defined ICT Pillars/Paradigms with all the four capabilities and are indicated on Table 2 with compliance index ' 4 '.

Considering the subsets: Sensing, Processing, Communication and Actuation capabilities that defined the ICT Pillars and Paradigms, innovative research in line with the current Pillars and Paradigms is seen as potential tool in disseminating pervasive healthcare. The compliance index of Robotics and Internet of Things technologies in relation to the four subsets that defined ICT Pillars and Paradigms which are also similar to the basic concept in communication engineering framework would enhance their suitability in ICT based healthcare system with little human attention.

Table 2. ICT pillars/paradigms, decomposed subsets and compliance index

\begin{tabular}{|c|c|c|c|c|c|c|}
\hline $\begin{array}{l}\text { ICT Pillars and } \\
\text { Paradigms }\end{array}$ & Abbreviations & Sensing & Processing & Communication & Actuation & Compliance Index \\
\hline Artificial Intelligence & $\mathrm{AI}$ & $\bullet$ & $\checkmark$ & $\bullet$ & $\bullet$ & 1 \\
\hline Big-Data Analytics & BDA & $\bullet$ & v & $\bullet$ & $\bullet$ & 1 \\
\hline Cognitive Computing & CG-C & $\bullet$ & v & $\bullet$ & $\bullet$ & 1 \\
\hline Soft Computing & SFT-C & $\bullet$ & v & $\bullet$ & $\bullet$ & 1 \\
\hline 3D Printing & 3D-P & $\bullet$ & $\bullet$ & $\bullet$ & v & 1 \\
\hline Cloud Computing & CLD-C & $\bullet$ & $\mathrm{v}$ & v & $\bullet$ & 2 \\
\hline FOG Computing & FOG-C & $\bullet$ & v & v & $\bullet$ & 2 \\
\hline Virtualization & VRT & $\bullet$ & v & v & $\bullet$ & 2 \\
\hline Wired Networks & Wd-Ns & $\bullet$ & $\bullet$ & v & $\bullet$ & 1 \\
\hline $\begin{array}{l}\text { Mobile Edge } \\
\text { Computing }\end{array}$ & ME-C & $\bullet$ & v & v & $\bullet$ & 2 \\
\hline Machine-to-Machine & M2M & $\bullet$ & $\bullet$ & v & $\bullet$ & 1 \\
\hline Robotics & RBT & v & v & v & v & 4 \\
\hline $\begin{array}{l}\text { Wireless Body Area } \\
\text { Networks }\end{array}$ & WBANs & $v$ & $\bullet$ & 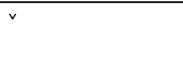 & $\bullet$ & 2 \\
\hline $\begin{array}{l}\text { Wireless Sensor } \\
\text { Networks }\end{array}$ & WSNs & v & $\bullet$ & v & $\bullet$ & 2 \\
\hline Internet of Things & IoT & v & v & v & v & 4 \\
\hline Social Media & SM & $\bullet$ & v & v & $\bullet$ & 2 \\
\hline Wireless Networks & Ws-Ns & $\bullet$ & $\bullet$ & v & $\bullet$ & 1 \\
\hline Smart Device & SD & v & $\bullet$ & $v^{2}$ & $\bullet$ & 2 \\
\hline Sensing Technologies & ST & v & $\bullet$ & $\bullet$ & $\bullet$ & 1 \\
\hline Mobile Cloud & $\mathrm{MC}$ & $\bullet$ & v & v & $\bullet$ & 2 \\
\hline Wearable Devices & WD & v & $\bullet$ & $\bullet$ & $\bullet$ & 1 \\
\hline
\end{tabular}

Source: Contents within the table are extracted from Google search engine

$\mathbf{v}$ (Indicate compliance with the subset defined per column)

- (Indicate non-compliance with the subset defined per column) 
Figure 4. Bar chart showing ICT pillars/paradigms compliance index in healthcare paradigms

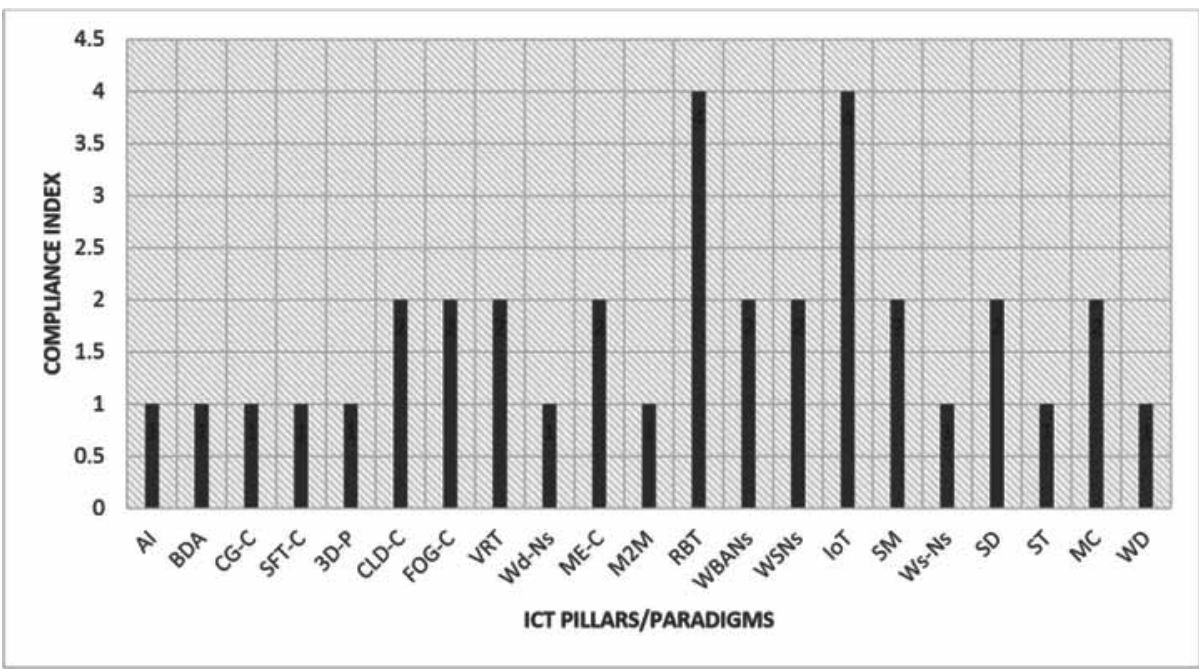

Figure 4 is a bar chart illustrating the level of compliance of each ICT Pillars/ Paradigms on Table 2 relative to the subsets that the ICT eco-system is decomposed.

Based on the compliance index illustrated on Figure 4, future innovations towards a pervasive healthcare(healthcare to anyone at anytime and anywhere) is anchored on robotics and IoT Paradigms. Therefore, addressing the inherent challenges that could abound in these technological innovations with high compliance index will enhance maximum profitability in the ICT based healthcare paradigms. Also, the hybrid of Pillars and Paradigms with low compliance index and those of high compliance index would yield a better innovation in providing pervasive healthcare since none of the technologies can operate effectively in isolation. Some technologies with lower compliance index, particularly; wearable, smart devices and sensing technologies can be integrated into and interoperate with IoT to cushion their performances.

\section{CONCLUSION}

Traditional healthcare cannot sufficiently accommodate the needs of the exponentially increasing human populace. Even though, there may be excellent medical infrastructure and cutting-edge technologies, medical services are not always affordable and available to everyone. Especially during global health issues such as the present Corona Virus Disease 2019 (COVID-19) pandemic that is ravaging the world. Based on ICT application trend and analysis in this paper, it is pertinent that technological innovative approaches are gradually transforming healthcare system and has the potential to re-position healthcare delivery from a reactive and hospital-centered to a proactive and patients' centered system; able to deliver preventive, predictive and corrective measures. The compliance level of technologies and paradigms such as: Robotics and Internet of Things to the subsets that defined the ICT eco-system 
enhances their suitability in providing pervasive healthcare. Therefore, adoption of technological advances in communication engineering is central to the establishment of pervasive healthcare system.

\section{RECOMMENDATIONS}

ICT based healthcare is a viable technological approach capable of providing support to healthcare professionals in addressing global healthcare concerns. However, innovative research towards pervasive healthcare demands may be confronted with challenging issues not particular to a single research area and therefore requires a collaborative research work from various fields of endeavor such as: medicine, engineering, social and economic sciences. Thus, in the implementation of technological innovations towards a pervasive healthcare system and to mitigate its multi-faced challenges, it must be ensured particularly of ICT Pillars/Paradigms with high level of compliance in terms of sensing, processing, communication and actuation that such system is:

1. Cost effective;

2. User and environmental friendly;

3. Energy efficient;

4. Bandwidth efficient;

5. Secured;

6. Robust and Scalable;

7. Inter- operational;

8. Responsive;

9. Accurate and operate at an acceptable speed;

10. Of quality service;

11. Of ample connectivity;

12. Popular to earn continuous support.

Future technological innovations and implementations is recommended in the areas of wireless sensor and actuator networks, wireless body area and actuator networks and full integration of Artificial Intelligence into healthcare domain. Pervasive healthcare system should be fully adopted in the treatment of communicable diseases and provision of healthcare services during viral outbreak like the corona virus to control its spread. 


\section{REFERENCES}

Bonomi, F., Milito, R., Zhu, J., \& Addepolli, S. (2012). Fog Computing and its role in the Internet of Things. In Proceedings of the first edition of the MCC workshop on Mobile Cloud Computing, (pp. 13-16). ACM doi:10.1145/2342509.2342513

Chen, M., Mao, S. \& Liu, Y. (2014). Big Data: A survey. Springer Science + Business Media.

Dhar, J., \& Ranganathan, A. (2015). Machine learning capabilities in medical diagnosis applications: Computational results for hepatitis disease. International Journal of Biomedical Engineering and Technology, 17(4), 330-340. doi:10.1504/ IJBET.2015.069398

Esteva, A., Kuprel, B., Novoa, R. A., Ko, J., Swetter, S. M., Blau, H. M., \& Thrun, S. (2017). Dermatologist-level classification of skin cancer with deep neural networks. Nature, 542(7638), 115-118. doi:10.1038/nature21056 PMID:28117445

Guiseppe, A., Valerio, P., \& Antonio, P. (2018). The role of Information and Communication Technologies in Healthcare: Taxonomy, Perspective and Challenges. Journal of Network and Computer Applications. Advance online publication. doi:10.1016/jnca2018.02.008

Mojtaba, A., Saeid, A., Mazdak, Z., Sabariah, B., \& Kouichi, S. (2016). Authentication in mobile cloud computing: A survey. Journal of Network and Computer Applications, 61, 59-80. doi:10.1016/j.jnca.2015.10.005

Myung, D., Duncan, B., Malan, D., Gaynor, M., \& Moulton, S. (2003). Vital Dust: Wireless Sensors and a Sensor Network for Real-time Patient Monitoring. Presented at the 8th Annual New England Regional Trauma conference, Burlington, MA.

Panda, M. (2014). Soft Computing: Concept and Techniques. University Science Press.

Polat, K., \& Gunes, S. (2008). Principle component analysis, fuzzy weighting pre-processing and artificial immune recognition system based diagnostic system for diagnosis of lung cancer. Expert Systems with Applications, 34(1), 214-221. doi:10.1016/j.eswa.2006.09.001

Prabha, S., Elias K., Saraju P.M. \&Madhavi K.G. (2017). Everything you wanted to know about smart health: Evaluating the different technologies and components of the Internet of Things for better health. IEEE Consumer Electronics Magazine. Doi:10.1109/MCE.2017.2755378

Rad, B. B., \& Shareef, A. A. (2017). Fog Computing: A Short Review of Concept and Applications. International Journal of Computer Science and Network Security, 17(11), 68-74.

Robotics. (n.d.). In Wikipedia. Retrieved May 18, 2020, from https:en.wikipedia.org/ wikiRobotics 
Sanael, Z., Abolfazli, S., Gani, A. \& Buyya, R. (2013). Heterogeneity in mobile cloud computing: Taxonomy and open challenges. IEEE Communication Survey and Tutorials, 1-24.

Shi, W., \& Schahram, D. (2016). The promise of Edge computing. Computer, 49, 78-81.

Shuo, T., Wembo, Y., Jehane, M.L.G., Peng, W., Wei, H. \&Zhewei, Y. (2019). Smart healthcare: making medical care more intelligent. Global Health Journal, 3(3), 62-65. http://<ALIGNMENT.qj>>/ALIGNMENT>10.1016/j.glohj.2019.07.001

Srinivas, J., Venkata, K., Reddy, S., \& Qyser, A. M. (2012). Cloud computing basics. International Journal of Advanced Research in Computer and Communication Engineering, 1(5), 2012.

Van-Kranenburg, R (2008). The internet of things.A critique of ambient technology and all-seeing network of RFID. Amsterdam: Institute of Network Cultures.

Varshney, U. (2007). Pervasive healthcare and wireless health monitoring. Mobile Networks and Applications, 12(2-3), 113-127. doi:10.1007/s11036-007-0017-1

Yingxu, W. (2009). On Cognitive Computing. International Journal of Software Science and Computational Intelligence, 1(3), 1-15. doi:10.4018/jssci.2009070101 
Oluwadara J. Odeyinka obtained his B.Tech in Electronic and Electrical Engineering from Ladoke Akintola University of Technology, Nigeria and M.Eng in Communication Engineering from Federal University of Technology, Owerri, Nigeria. His research interest is in the area of Wireless Sensor Networks and Machine learning.

Opeyemi A. Ajibola received BTech (Hons) in Electronic and Electrical Engineering from Ladoke Akintola University of Technology, Ogbomoso, Nigeria in 2011 and MSc in Electrical and Electronic Engineering with specialization in Communication Engineering from University of Lagos, Nigeria in 2016 respectively. He is currently the acting HOD, Department of Electrical and Telecommunication Engineering, ESGT - Benin University, Republic of Benin. His research interest is in the area of Wireless Communication, Digital Signal Processing and Machine Learning.

Michael C. Ndinechi was the formal Director, ICT Center and he is currently the Dean, School of Electrical System Engineering Technology, Federal University of Technology, (FUT) Owerri, Nigeria. He has served as Head of Department of Electrical and Electronic Engineering (2015 to 2016) and Associate Dean of the School of Engineering and Engineering Technology FUT, Owerri (2008 to 2010). He was the Director/CEO of the Electronics Development Institute, ELDI, Awka, Federal Ministry of Science and Technology, Nigeria from 2010 to 2014. He obtained his PhD degree in 2008 and specialized in Electrical and Electronics Engineering. In 1995, he obtained the University of Greenwich, London postgraduate certificate in Data Communications. He is registered with the Council for the Regulation of Engineering in Nigeria (COREN), a Corporate Member of Nigerian Society of Engineers, and a Member of Nigerian Institute of Management. Prof. Ndinechi has received many awards which include Federal Government of Nigeria Postgraduate Scholarship Award 1990, World Bank/National Universities Commission (Nigeria) Research Fellowship Award, 1994, Africa Educational Trust Award, London, 1995, National Award for Industrial and Business Development (NAIBD), Distinguished Leadership in National Development Gold Award (D'LINGA 2012) etc. His research interest is on Algorithms for Energy Conservation in Digital Systems, Modeling and Simulation of Wireless Sensor Networks using IoT. 